\title{
Simple and efficient representations for the fundamental solutions of Stokes flow in a half-space
}

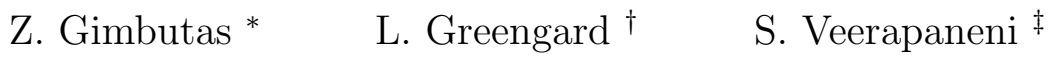

October 17,2018

\begin{abstract}
We derive new formulas for the fundamental solutions of slow, viscous flow, governed by the Stokes equations, in a half-space. They are simpler than the classical representations obtained by Blake and collaborators, and can be efficiently implemented using existing fast solvers libraries. We show, for example, that the velocity field induced by a Stokeslet can be annihilated on the boundary (to establish a zero slip condition) using a single reflected Stokeslet combined with a single Papkovich-Neuber potential that involves only a scalar harmonic function. The new representation has a physically intuitive interpretation.
\end{abstract}

\section{Introduction}

Viscous flow of passive and active suspensions in the presence of an infinite planar boundary is an important physical model in many areas of science and engineering. It serves as a useful paradigm for understanding the effect of confined geometries on the macroscopic flow behavior of particulate flows, for example, that of bacterial propulsion, cellular blood flow and colloidal suspensions, [15, 7, 3, 23, 21]. In problems where the Reynolds number is low, the ambient fluid is governed by the Stokes equations:

$$
\mu \Delta \mathbf{u}(\mathbf{x})=\nabla p(\mathbf{x}), \quad \nabla \cdot \mathbf{u}(\mathbf{x})=0,
$$

where $\mu$ is the fluid viscosity, $\mathbf{u}(\mathbf{x})=\left(u_{1}(\mathbf{x}), u_{2}(\mathbf{x}), u_{3}(\mathbf{x})\right)$ is the velocity of the fluid, and $p(\mathbf{x})$ is the pressure. Assuming the plane wall is located at $x_{3}=0$ and that the flow velocity decays in the far field,

$$
\mathbf{u}(\mathbf{x}) \rightarrow 0, \quad \text { as } \quad|\mathbf{x}| \rightarrow \infty
$$

\footnotetext{
*Information Technology Laboratory, National Institute of Standards and Technology, 325 Broadway, Mail Stop 891.01, Boulder, CO 80305-3328. email: zydrunas.gimbutas@nist.gov. The work of this author was supported in part by the Office of the Assistant Secretary of Defense for Research and Engineering and AFOSR under NSSEFF Program Award FA9550-10-1-0180. and in part by the National Science Foundation under grant DMS-0934733. Contributions by staff of NIST, an agency of the U.S. Government, are not subject to copyright within the United States.

${ }^{\dagger}$ Simons Center for Data Analysis, Simons Foundation, 160 Fifth Avenue, NY, NY 10010 and Courant Institute of Mathematical Sciences, New York University, 251 Mercer Street, New York, NY 10012-1110. email: greengard@cims.nyu.edu. The work of this author was supported in part by the Office of the Assistant Secretary of Defense for Research and Engineering and AFOSR under NSSEFF Program Award FA9550-10-1-0180, by the National Science Foundation under grant DMS-0934733, and by the Applied Mathematical Sciences Program of the U.S. Department of Energy under Contract DEFGO288ER25053.

${ }^{\ddagger}$ Department of Mathematics, University of Michigan, 530 Church Street, Ann Arbor, MI 48109. email: shravan@umich.edu. The work of this author was supported by the NSF under grants DMS-1418964 and DMS-1224656.
} 
the no-slip boundary condition is

$$
\left.\mathbf{u}\right|_{x_{3}=0}=0 .
$$

Boundary integral methods are particularly well-suited for problems of this kind since they discretize the domain boundary alone (resulting in many fewer degrees of freedom) and impose the decay and the no-slip conditions exactly. Moreover, they avoid the need for artificial truncation of the computational domain and can be solved rapidly and with great accuracy using fast algorithms such as the fast multipole method (FMM) and high order accurate quadrature rules.

In order to reformulate the Stokes equations as a boundary integral equation, however, one needs to have access to the Green's functions for the half-space [16, 14]. A now classical approach to constructing this Green's function is due to Blake and others [4, 5, 6, 1, 2, 20, 29]. Unfortunately, the resulting formulas are rather complicated, making them somewhat difficult to implement.

Here, we show that a much simpler alternative to the Blake solution can be obtained by combining a free space image, which annihilates the tangential components of velocity, with a PapkovichNeuber potential [19, 18] which annihilates the normal component.

The present paper is organized as follows. We discuss Papkovich-Neuber potentials and the standard fundamental solutions for the Stokes equations in section 2, (See, for example, [16, 14, [15, 20]). We also review Blake's formula for the Stokeslet in a half-space. In section 3, we derive the image structures for Stokeslets, stresslets, rotlets, and Stokes doublets.

In the appendices, we provide the analogous formulas for two-dimensional half-space Stokes kernels and for some problems of linear elasticity.

\section{Fundamental solutions, the Papkovich-Neuber representation, and Blake's formulas}

Before discussing the various standard fundamental solutions for the Stokes equations in free-space, we introduce the Papkovich-Neuber representation, originally developed in [19, 18] for problems of linear elasticity. Without loss of generality, we assume that the fluid viscosity $\mu=1$ in the rest of the paper.

Definition 2.1. Let $\phi(\boldsymbol{x}), \boldsymbol{x} \in \mathbb{R}^{3}$ be a harmonic function. Then the induced Papkovich-Neuber representation is defined to be the paired vector field $\mathbf{u}$ and scalar field $p$ given by:

$$
\mathbf{u}(\boldsymbol{x})=x_{3} \nabla \phi(\boldsymbol{x})-\left[\begin{array}{c}
0 \\
0 \\
\phi(\boldsymbol{x})
\end{array}\right], \quad p(\boldsymbol{x})=2 \frac{\partial \phi(\boldsymbol{x})}{\partial x_{3}} .
$$

It is straightforward to verify that $(\mathbf{u}, p)$ satisfy the Stokes equations (1) (with $\mu=1$ ).

Suppose now that a force vector $\mathbf{f}=\left(f_{1}, f_{2}, f_{3}\right)$ is applied to a viscous fluid at a point $\mathbf{y}$. Then, it is well-known that the induced velocity and pressure can be computed using the Stokeslet (the single layer kernel):

$$
\begin{gathered}
S_{i j}(\mathbf{x}, \mathbf{y})=\frac{1}{8 \pi}\left[\frac{\delta_{i j}}{|\mathbf{x}-\mathbf{y}|}+\frac{\left(x_{i}-y_{i}\right)\left(x_{j}-y_{j}\right)}{|\mathbf{x}-\mathbf{y}|^{3}}\right], \quad i, j=1,2,3 \\
P_{j}(\mathbf{x}, \mathbf{y})=\frac{1}{4 \pi} \frac{x_{j}-y_{j}}{|\mathbf{x}-\mathbf{y}|^{3}}
\end{gathered}
$$


where $\delta_{i j}$ is the Kronecker delta. More precisely, the velocity vector $\mathbf{u}(\mathbf{x})$ and pressure $p(\mathbf{x})$ are given by

$$
u_{i}(\mathbf{x})=\sum_{j=1}^{3} S_{i j}(\mathbf{x}, \mathbf{y}) f_{j}, \quad p(\mathbf{x})=\sum_{j=1}^{3} P_{j}(\mathbf{x}, \mathbf{y}) f_{j} .
$$

The stresslet (or double layer kernel) for the Stokes equations describes the velocity induced by an infinitesimal displacement $\mathbf{g}=\left(g_{1}, g_{2}, g_{3}\right)$ (sometimes called the double force source strength) at a point $\mathbf{y}$ with orientation vector $\boldsymbol{\nu}=\left(\nu_{1}, \nu_{2}, \nu_{3}\right)$. It is given by:

$$
\begin{gathered}
T_{i j k}(\mathbf{x}, \mathbf{y})=\frac{3}{4 \pi} \frac{\left(x_{i}-y_{i}\right)\left(x_{j}-y_{j}\right)\left(x_{k}-y_{k}\right)}{|\mathbf{x}-\mathbf{y}|^{5}}, \quad i, j, k=1,2,3, \\
\Pi_{j k}(\mathbf{x}, \mathbf{y})=\frac{1}{2 \pi}\left[-\frac{\delta_{j k}}{|\mathbf{x}-\mathbf{y}|^{3}}+\frac{3\left(x_{j}-y_{j}\right)\left(x_{k}-y_{k}\right)}{|\mathbf{x}-\mathbf{y}|^{5}}\right],
\end{gathered}
$$

and the corresponding formulas for the velocity and pressure at an arbitrary point $\mathbf{x}$ are

$$
u_{i}(\mathbf{x})=\sum_{j=1}^{3} \sum_{k=1}^{3} T_{i j k}(\mathbf{x}, \mathbf{y}) \nu_{k} g_{j}, \quad p(\mathbf{x})=\sum_{j=1}^{3} \sum_{k=1}^{3} \Pi_{j k}(\mathbf{x}, \mathbf{y}) \nu_{k} g_{j}
$$

\subsection{Stokes flow in a half-space, Blake's formula}

Suppose now that a force vector $\mathbf{f}$ is applied to a viscous fluid in the upper half-space $\left(x_{3}>0\right)$. Then the corresponding Stokeslet-induced velocity field fails to satisfy the no-slip condition (2). In order to annihilate the velocity field while satisfying the homogeneous Stokes equations in the upper half-space, 4] proposed the following image structure:

$$
S_{i j}^{W}(\mathbf{x}, \mathbf{y})=S_{i j}(\mathbf{x}, \mathbf{y})-S_{i j}\left(\mathbf{x}, \mathbf{y}^{I}\right)+2 y_{3}^{2} S_{i j}^{D}\left(\mathbf{x}, \mathbf{y}^{I}\right)-2 y_{3} S_{i j}^{S D}\left(\mathbf{x}, \mathbf{y}^{I}\right)
$$

where $\mathbf{y}^{I}=\left(y_{1}, y_{2},-y_{3}\right)$ is the reflected image location. Here, $S^{D}$ is a modified source doublet given by

$$
S_{i j}^{D}(\mathbf{x}, \mathbf{y})=\frac{1}{8 \pi}\left(1-2 \delta_{j 3}\right) \frac{\partial}{\partial x_{j}} \frac{x_{i}-y_{i}}{|\mathbf{x}-\mathbf{y}|^{3}}
$$

and $S^{S D}$ is a modified Stokes doublet, given by

$$
S_{i j}^{S D}(\mathbf{x}, \mathbf{y})=\left(1-2 \delta_{j 3}\right) \frac{\partial S_{i 3}(\mathbf{x}, \mathbf{y})}{\partial x_{j}} .
$$

Similar, but more involved, decompositions for the Stokes doublet and stresslet in a half-space are given in [6] and [20]. Note that the computation of the modified source doublet $S_{i j}^{D}$ requires the evaluation of three distinct harmonic dipole fields.

\section{A new image formula}

In this section, we derive a simpler image structure, using the Papkovich-Neuber representation which involves only a single harmonic function. $\mathbf{y}^{I}$, as above, will denote the image location 
$\left(y_{1}, y_{2},-y_{3}\right)$. Now, however, we define reflected single force, double force, and double force orientation vectors by negating their third components:

$$
\mathbf{f}^{I}=\left(f_{1}, f_{2},-f_{3}\right), \quad \mathbf{g}^{I}=\left(g_{1}, g_{2},-g_{3}\right), \quad \boldsymbol{\nu}^{I}=\left(\nu_{1}, \nu_{2},-\nu_{3}\right) .
$$

We also make use of the harmonic potential due to a unit strength charge,

$$
G^{S}(\mathbf{x}, \mathbf{y})=\frac{1}{4 \pi|\mathbf{x}-\mathbf{y}|}
$$

the harmonic potential due to a unit strength dipole with orientation vector $\boldsymbol{\nu}$,

$$
G^{D}[\boldsymbol{\nu}](\mathbf{x}, \mathbf{y})=\sum_{i=1}^{3} \nu_{i} \frac{\partial}{\partial y_{i}} \frac{1}{4 \pi|\mathbf{x}-\mathbf{y}|},
$$

and the harmonic potential due to a unit strength quadrupole with orientation vectors $\boldsymbol{\nu}$ and $\boldsymbol{\kappa}$,

$$
G^{Q}[\boldsymbol{\nu}, \boldsymbol{\kappa}](\mathbf{x}, \mathbf{y})=\sum_{i=1}^{3} \sum_{j=1}^{3} \nu_{i} \kappa_{j} \frac{\partial^{2}}{\partial y_{i} \partial y_{j}} \frac{1}{4 \pi|\mathbf{x}-\mathbf{y}|}
$$

Finally, recall that, through the Papkovich-Neuber representation (3), the harmonic function $\phi(\mathbf{x})$ induces velocity and pressure fields that can be written in component form as

$$
u_{i}(\mathbf{x})=x_{3} \frac{\partial}{\partial x_{i}} \phi(\mathbf{x})-\delta_{i 3} \phi(\mathbf{x}), \quad p(\mathbf{x})=2 \frac{\partial}{\partial x_{3}} \phi(\mathbf{x}) .
$$

Of particular note is the fact that at $x_{3}=0$, the only non-zero velocity component is

$$
u_{3}(\mathbf{x})=-\phi(\mathbf{x}) .
$$

This suggests a simple two-step strategy. First, annihilate the tangential components of the velocity field induced by a Stokeslet (single layer) or stresslet (double layer) kernel. It is easy to see that this can be accomplished by subtracting the influence of a reflected single force $\mathbf{f}^{I}=$ $\left(f_{1}, f_{2},-f_{3}\right)$, or double force $\mathbf{g}^{I}=\left(g_{1}, g_{2},-g_{3}\right), \boldsymbol{\nu}^{I}=\left(\nu_{1}, \nu_{2},-\nu_{3}\right)$ located at the image point $\mathbf{y}^{I}$, respectively. It remains only to match the remaining non-zero normal component $u_{3}$, which we will do by a judicious choice of the Papkovich-Neuber potential $\phi(\mathbf{x})$.

\subsection{The Stokeslet correction}

Following the discussion above, let us write out in more detail the velocity field $\mathbf{v}$ remaining after subtracting the image Stokeslet at $\mathbf{y}^{I}$ from the original Stokeslet at $\mathbf{y}$ (Fig. 1):

$$
v_{i}(\mathbf{x})=\sum_{j=1}^{3} S_{i j}(\mathbf{x}, \mathbf{y}) f_{j}-\sum_{j=1}^{3} S_{i j}\left(\mathbf{x}, \mathbf{y}^{I}\right) f_{j}^{I} .
$$

At the interface $x_{3}=0$, a straightforward computation yields

$$
v_{1}(\mathbf{x})=0, \quad v_{2}(\mathbf{x})=0, \quad v_{3}(\mathbf{x})=-\frac{f_{3}^{I}}{4 \pi} \frac{1}{\left|\mathbf{x}-\mathbf{y}^{I}\right|}-\frac{y_{3}}{4 \pi} \sum_{j=1}^{3} \frac{x_{j}-y_{j}^{I}}{\left|\mathbf{x}-\mathbf{y}^{I}\right|^{3}} f_{j}^{I}
$$




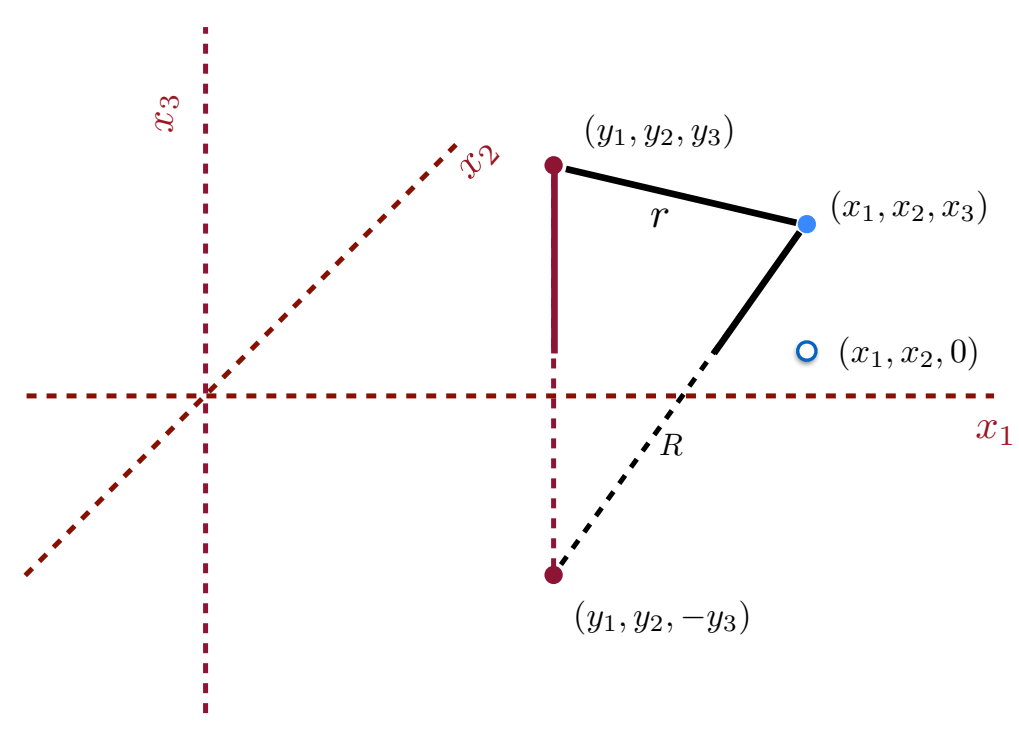

Figure 1: The original source location is in the upper half-space at $\left(y_{1}, y_{2}, y_{3}\right)$ and the reflected image source is at $\left(y_{1}, y_{2},-y_{3}\right)$. For target points $\left(x_{1}, x_{2}, 0\right)$ that lie on the half-space boundary, the distances $r$ and $R$ from the source and image are the same, simplifying the computation of $\mathbf{v}$ in 20$)$.

By inspection of $(20)$, it is clear that the harmonic potential $\phi$ required to cancel the non-zero normal component $v_{3}$ at the interface is that induced by a charge of strength $f_{3}^{I}$ and a dipole with orientation vector $\mathbf{f}^{I}$ of strength $y_{3}$ located at $\mathbf{y}^{I}$. Thus, the velocity field $\mathbf{u}^{W}$ satisfying the desired no-slip boundary condition (2) can be expressed as:

$$
u_{i}^{W}(\mathbf{x})=u_{i}^{A}(\mathbf{x})-u_{i}^{B}(\mathbf{x})-u_{i}^{C}(\mathbf{x}),
$$

where $\mathbf{u}^{A}$ and $\mathbf{u}^{B}$ are the velocity fields induced by the original free-space Stokeslet and the reflected image force vector, respectively:

$$
u_{i}^{A}(\mathbf{x})=\sum_{j=1}^{3} S_{i j}(\mathbf{x}, \mathbf{y}) f_{j}, \quad u_{i}^{B}(\mathbf{x})=\sum_{j=1}^{3} S_{i j}\left(\mathbf{x}, \mathbf{y}^{I}\right) f_{j}^{I} .
$$

Here, $u_{i}^{C}$ is the Papkovich-Neuber correction

$$
u_{i}^{C}(\mathbf{x})=x_{3} \frac{\partial}{\partial x_{i}} \phi(\mathbf{x})-\delta_{i 3} \phi(\mathbf{x}),
$$

where the harmonic potential $\phi$ is that due to a simple charge and dipole, both located at $\mathbf{y}^{I}$ :

$$
\phi(\mathbf{x})=f_{3}^{I} G^{S}\left(\mathbf{x}, \mathbf{y}^{I}\right)+y_{3} G^{D}\left[\mathbf{f}^{I}\right]\left(\mathbf{x}, \mathbf{y}^{I}\right) .
$$

\subsection{The stresslet correction}

We state the image structure for the stresslet (the double layer kernel) in the form of a theorem. 
Theorem 1. Let a double force $\mathbf{g}$ with orientation vector $\boldsymbol{\nu}$ be located at $\boldsymbol{y}$, resulting in the free-space velocity field

$$
u_{i}^{T}(\boldsymbol{x})=\sum_{j=1}^{3} \sum_{k=1}^{3} T_{i j k}(\boldsymbol{x}, \boldsymbol{y}) \nu_{k} g_{j}
$$

Then the corresponding velocity satisfying the no-slip boundary condition (2) is given by

$$
u_{i}^{T, W}(\boldsymbol{x})=\sum_{j=1}^{3} \sum_{k=1}^{3} T_{i j k}(\boldsymbol{x}, \boldsymbol{y}) \nu_{k} g_{j}-\sum_{j=1}^{3} \sum_{k=1}^{3} T_{i j k}\left(\boldsymbol{x}, \boldsymbol{y}^{I}\right) \nu_{k}^{I} g_{j}^{I}-\left[x_{3} \frac{\partial}{\partial x_{i}} \phi^{T}(\boldsymbol{x})-\delta_{i 3} \phi^{T}(\boldsymbol{x})\right]
$$

where

$$
\phi^{T}(\boldsymbol{x})=2\left(\boldsymbol{\nu}^{I} \cdot \mathbf{g}^{I}\right) G^{D}[\mathbf{h}]\left(\boldsymbol{x}, \boldsymbol{y}^{I}\right)+2 y_{3} G^{Q}\left[\boldsymbol{\nu}^{I}, \mathbf{g}^{I}\right]\left(\boldsymbol{x}, \boldsymbol{y}^{I}\right)
$$

with $\mathbf{h}=(0,0,1)$.

Proof. Note first that the symmetric part of the Stokes doublet is given by

$$
T_{i j k}^{S}(\mathbf{x}, \mathbf{y})=\frac{1}{4 \pi}\left[-\frac{\left(x_{i}-y_{i}\right)}{|\mathbf{x}-\mathbf{y}|^{3}} \delta_{j k}+\frac{3\left(x_{i}-y_{i}\right)\left(x_{j}-y_{j}\right)\left(x_{k}-y_{k}\right)}{|\mathbf{x}-\mathbf{y}|^{5}}\right] .
$$

Furthermore, the tangential components of the velocity field

$$
v_{i}(\mathbf{x})=\sum_{j=1}^{3} \sum_{k=1}^{3} T_{i j k}^{S}(\mathbf{x}, \mathbf{y}) \nu_{k} g_{j}-\sum_{j=1}^{3} \sum_{k=1}^{3} T_{i j k}^{S}\left(\mathbf{x}, \mathbf{y}^{I}\right) \nu_{k}^{I} g_{j}^{I}
$$

are zero when $x_{3}=0$. Thus, the harmonic potential $\phi^{S}$ annihilating $v_{3}$ at the interface $x_{3}=0$ is that due to a single quadrupole source located at $\mathbf{y}^{I}$ with orientation vectors $\boldsymbol{\nu}^{I}, \mathbf{g}^{I}$ and strength $2 y_{3}$ :

$$
\phi^{S}(\mathbf{x})=2 y_{3} G^{Q}\left[\boldsymbol{\nu}^{I}, \mathbf{g}^{I}\right]\left(\mathbf{x}, \mathbf{y}^{I}\right) .
$$

It is straightforward to check that the stresslet is simply related to the symmetric part of the Stokes doublet

$$
T_{i j k}(\mathbf{x}, \mathbf{y})=\frac{3}{4 \pi} \frac{\left(x_{i}-y_{i}\right)\left(x_{j}-y_{j}\right)\left(x_{k}-y_{k}\right)}{|\mathbf{x}-\mathbf{y}|^{5}}=\frac{1}{4 \pi} \frac{x_{i}-y_{i}}{|\mathbf{x}-\mathbf{y}|^{3}} \delta_{j k}+T_{i j k}^{S}(\mathbf{x}, \mathbf{y}) .
$$

Thus, it remains only to annihilate the velocity field induced by the first term on the right-hand side of (31) and its reflected image, given by

$$
v_{i}(\mathbf{x})=\frac{1}{4 \pi} \sum_{j=1}^{3} \sum_{k=1}^{3} \frac{x_{i}-y_{i}}{|\mathbf{x}-\mathbf{y}|^{3}} \delta_{j k} \nu_{k} g_{j}-\frac{1}{4 \pi} \sum_{j=1}^{3} \sum_{k=1}^{3} \frac{x_{i}-y_{i}^{I}}{\left|\mathbf{x}-\mathbf{y}^{I}\right|^{3}} \delta_{j k} \nu_{k}^{I} g_{j}^{I} .
$$

At $x_{3}=0$, the tangential components vanish and the normal component is easily computed to be $2\left(\boldsymbol{\nu}^{I} \cdot \mathbf{g}^{I}\right) G^{D}[\mathbf{h}]\left(\mathbf{x}, \mathbf{y}^{I}\right)$, where $\mathbf{h}=(0,0,1)$. The desired result follows. 


\subsection{The rotlet correction}

Similar representations are easily derived for other fundamental solutions, such as the rotlet - the antisymmetric part of the Stokes doublet:

$$
T_{i j k}^{R}(\mathbf{x}, \mathbf{y})=\frac{1}{4 \pi}\left[-\frac{\left(x_{j}-y_{j}\right)}{|\mathbf{x}-\mathbf{y}|^{3}} \delta_{i k}+\frac{\left(x_{k}-y_{k}\right)}{|\mathbf{x}-\mathbf{y}|^{3}} \delta_{i j}\right] .
$$

The corresponding velocity satisfying the no-slip boundary condition (2) is given by

$$
u_{i}^{R, W}(\mathbf{x})=\sum_{j=1}^{3} \sum_{k=1}^{3} T_{i j k}^{R}(\mathbf{x}, \mathbf{y}) \nu_{k} g_{j}-\sum_{j=1}^{3} \sum_{k=1}^{3} T_{i j k}^{R}\left(\mathbf{x}, \mathbf{y}^{I}\right) \nu_{k}^{I} g_{j}^{I}-\left[x_{3} \frac{\partial}{\partial x_{i}} \phi^{R}(\mathbf{x})-\delta_{i 3} \phi^{R}(\mathbf{x})\right],
$$

where the Papkovich-Neuber correction $\phi^{R}$ is due to two dipoles:

$$
\phi^{R}(\mathbf{x})=-2 \nu_{3}^{I} G^{D}\left[\mathbf{g}^{I}\right]\left(\mathbf{x}, \mathbf{y}^{I}\right)+2 g_{3}^{I} G^{D}\left[\boldsymbol{\nu}^{I}\right]\left(\mathbf{x}, \mathbf{y}^{I}\right)
$$

\subsection{The Stokes doublet correction}

Finally, the Stokes doublet is the sum of its symmetric and antisymmetric parts

$$
\begin{aligned}
T_{i j k}^{D}(\mathbf{x}, \mathbf{y}) & =\frac{1}{4 \pi}\left[-\frac{\left(x_{i}-y_{i}\right)}{|\mathbf{x}-\mathbf{y}|^{3}} \delta_{j k}-\frac{\left(x_{j}-y_{j}\right)}{|\mathbf{x}-\mathbf{y}|^{3}} \delta_{i k}+\frac{\left(x_{k}-y_{k}\right)}{|\mathbf{x}-\mathbf{y}|^{3}} \delta_{i j}\right. \\
& \left.+\frac{3\left(x_{i}-y_{i}\right)\left(x_{j}-y_{j}\right)\left(x_{k}-y_{k}\right)}{|\mathbf{x}-\mathbf{y}|^{5}}\right]=T_{i j k}^{S}(\mathbf{x}, \mathbf{y})+T_{i j k}^{R}(\mathbf{x}, \mathbf{y}) .
\end{aligned}
$$

By combining (30) and (35), we obtain the image formula for the Stokes doublet

$$
\begin{aligned}
u_{i}^{D, W}(\mathbf{x}) & =\sum_{j=1}^{3} \sum_{k=1}^{3} T_{i j k}^{D}(\mathbf{x}, \mathbf{y}) \nu_{k} g_{j}-\sum_{j=1}^{3} \sum_{k=1}^{3} T_{i j k}^{D}\left(\mathbf{x}, \mathbf{y}^{I}\right) \nu_{k}^{I} g_{j}^{I} \\
& -\left[x_{3} \frac{\partial}{\partial x_{i}} \phi^{D}(\mathbf{x})-\delta_{i 3} \phi^{D}(\mathbf{x})\right],
\end{aligned}
$$

where the Papkovich-Neuber potential is given by

$$
\phi^{D}(\mathbf{x})=-2 \nu_{3}^{I} G^{D}\left[\mathbf{g}^{I}\right]\left(\mathbf{x}, \mathbf{y}^{I}\right)+2 g_{3}^{I} G^{D}\left[\boldsymbol{\nu}^{I}\right]\left(\mathbf{x}, \mathbf{y}^{I}\right)+2 y_{3} G^{Q}\left[\boldsymbol{\nu}^{I}, \mathbf{g}^{I}\right]\left(\mathbf{x}, \mathbf{y}^{I}\right) .
$$

\section{Conclusions}

We have derived very simple image formulas for Stokes flow in a half-space induced by any of the standard fundamental solutions - the Stokeslet, stresslet, rotlet, and Stokes doublet. In each case, all that is required is a reflected fundamental solution and a Papkovich-Neuber correction based on a single harmonic potential.

The velocity (and pressure) due to the "direct" and reflected fundamental solutions can be computed together with any software that handles Stokeslets, stresslets, etc. in free space. Furthermore, the Papkovich-Neuber potential requires only the evaluation of a single additional harmonic function - itself requiring only software for free space harmonic sources, dipoles and quadrupoles. Many efficient schemes exist for these various steps, such as those described in [10, 11, 28, 27, 9, 25, 13, 26, 24, 22, 8, 12]. 


\section{A Extension to the two-dimensional problems}

The two-dimensional Stokes flow representations in a half-space can be derived similarly. They lead to identical Papkovich-Neuber corrections with corresponding charge, dipole, and quadrupole potentials replaced by their two-dimensional equivalents. Similar to the three-dimensional case, for any $\mathbf{x} \in \mathbb{R}^{2}$, the velocity field $\mathbf{u}^{W}$ satisfying the no-slip boundary condition in a half-plane is composed of three terms:

$$
\mathbf{u}^{W}(\mathbf{x})=\mathbf{u}^{A}(\mathbf{x})-\mathbf{u}^{B}(\mathbf{x})-\mathbf{u}^{C}(\mathbf{x}),
$$

where the first term is the velocity field induced by the free-space Green's function, the second term is the reflected image about the plane wall annihilating the tangential velocity component, and the third term is a Papkovich-Neuber correction term in the following form:

$$
\mathbf{u}^{C}(\mathbf{x})=x_{2} \nabla \phi(\mathbf{x})-\left[\begin{array}{c}
0 \\
\phi(\mathbf{x})
\end{array}\right], \quad p(\mathbf{x})=2 \frac{\partial \phi(\mathbf{x})}{\partial x_{2}} .
$$

The correction potentials for various fundamental solutions are

$$
\phi(\mathbf{x})=f_{2}^{I} G^{S}\left(\mathbf{x}, \mathbf{y}^{I}\right)+y_{2} G^{D}\left[\mathbf{f}^{I}\right]\left(\mathbf{x}, \mathbf{y}^{I}\right),
$$

for the two-dimensional Stokeslet, and

$$
\begin{gathered}
\phi^{T}(\mathbf{x})=2\left(\boldsymbol{\nu}^{I} \cdot \mathbf{g}^{I}\right) G^{D}[\mathbf{h}]\left(\mathbf{x}, \mathbf{y}^{I}\right)+2 y_{2} G^{Q}\left[\boldsymbol{\nu}^{I}, \mathbf{g}^{I}\right]\left(\mathbf{x}, \mathbf{y}^{I}\right), \\
\phi^{R}(\mathbf{x})=-2 \nu_{2}^{I} G^{D}\left[\mathbf{g}^{I}\right]\left(\mathbf{x}, \mathbf{y}^{I}\right)+2 \mathbf{g}_{2}^{I} G^{D}\left[\boldsymbol{\nu}^{I}\right]\left(\mathbf{x}, \mathbf{y}^{I}\right), \\
\phi^{D}(\mathbf{x})=-2 \nu_{2}^{I} G^{D}\left[\mathbf{g}^{I}\right]\left(\mathbf{x}, \mathbf{y}^{I}\right)+2 g_{2}^{I} G^{D}\left[\boldsymbol{\nu}^{I}\right]\left(\mathbf{x}, \mathbf{y}^{I}\right)+2 y_{2} G^{Q}\left[\boldsymbol{\nu}^{I}, \mathbf{g}^{I}\right]\left(\mathbf{x}, \mathbf{y}^{I}\right),
\end{gathered}
$$

for the two-dimensional stresslet, rotlet, and Stokes doublet, respectively, where the images of the source, single force, double force, and double force orientation vectors are

$$
\mathbf{y}^{I}=\left(y_{1},-y_{2}\right), \quad \mathbf{f}^{I}=\left(f_{1},-f_{2}\right), \quad \mathbf{g}^{I}=\left(g_{1},-g_{2}\right), \quad \boldsymbol{\nu}^{I}=\left(\nu_{1},-\nu_{2}\right),
$$

with the orientation vector $\mathbf{h}=(0,1)$, and the free-space Laplace Green's functions in two dimensions are given by

$$
\begin{gathered}
G^{S}(\mathbf{x}, \mathbf{y})=-\frac{1}{2 \pi} \log |\mathbf{x}-\mathbf{y}|, \quad G^{D}[\boldsymbol{\nu}](\mathbf{x}, \mathbf{y})=\sum_{i=1}^{2} \nu_{i} \frac{\partial}{\partial y_{i}} G^{S}(\mathbf{x}, \mathbf{y}) \\
\text { and } G^{Q}[\boldsymbol{\nu}, \boldsymbol{\kappa}](\mathbf{x}, \mathbf{y})=\sum_{i=1}^{2} \sum_{j=1}^{2} \nu_{i} \kappa_{j} \frac{\partial^{2}}{\partial y_{i} \partial y_{j}} G^{S}(\mathbf{x}, \mathbf{y}) .
\end{gathered}
$$

\section{B Extension to linear elasticity kernels}

The single layer kernel for linear isotropic elasticity in $R^{3}$ is given by Kelvin's solution [17]

$$
U_{i j}(\mathbf{x}, \mathbf{y})=\frac{1}{8 \pi \mu}\left[(2-\alpha) \frac{\delta_{i j}}{|\mathbf{x}-\mathbf{y}|}+\alpha \frac{\left(x_{i}-y_{i}\right)\left(x_{j}-y_{j}\right)}{|\mathbf{x}-\mathbf{y}|^{3}}\right], \quad i, j=1,2,3,
$$


where $\lambda, \mu$ are Lame's parameters and $\alpha=(\lambda+\mu) /(\lambda+2 \mu)$. It is easy to see that the displacement field

$$
u_{i}(\mathbf{x})=\sum_{j=1}^{3} S_{i j}(\mathbf{x}, \mathbf{y}) f_{j}-\sum_{j=1}^{3} S_{i j}\left(\mathbf{x}, \mathbf{y}^{I}\right) f_{j}^{I}-u_{i}^{C}(\mathbf{x})
$$

satisfies the no-displacement boundary condition $\mathbf{u}(\mathbf{x})=0$ at $x_{3}=0$, where the Papkovich-Neuber correction is

$$
\begin{aligned}
& u_{i}^{C}(\mathbf{x})=\alpha x_{3} \frac{\partial}{\partial x_{i}} \phi(\mathbf{x})-(2-\alpha) \delta_{i 3} \phi(\mathbf{x}), \\
& \phi(\mathbf{x})=\frac{f_{3}^{I}}{\mu} G^{S}\left(\mathbf{x}, \mathbf{y}^{I}\right)+\frac{y_{3}}{\mu} G^{D}\left[\mathbf{f}^{I}\right]\left(\mathbf{x}, \mathbf{y}^{I}\right) .
\end{aligned}
$$

\section{References}

[1] K. Aderogba and J. R. Blake. Action of a force near the planar surface between semi-infinite immiscible liquids at very low Reynolds numbers: Addendum. Bulletin of the Australian Mathematical Society, 19(2):309-318, 1978.

[2] K. Aderogba and J. R. Blake. Action of a force near the planar surface between two semi-infinite immiscible liquids at very low Reynolds numbers. Bulletin of the Australian Mathematical Society, 18(3):345-356, 1978.

[3] S. Bhattacharya and J. Bławzdziewicz. Image system for Stokes-flow singularity between two parallel planar walls. Journal of Mathematical Physics, 43(11):5720-5731, 2002.

[4] J. R. Blake. A note on the image system for a Stokeslet in a no-slip boundary. Mathematical Proceedings of the Cambridge Philosophical Society, 70(2):303-310, 1971.

[5] J. R. Blake. Singularities of viscous flow. Journal of Engineering Mathematics, 8(2):113-124, 1974 .

[6] J. R. Blake and A. T. Chwang. Fundamental singularities of viscous flow. Journal of Engineering Mathematics, 8(1):23-29, 1974.

[7] B. Cichocki and R. B. Jones. Image representation of a spherical particle near a hard wall. Physica A: Statistical Mechanics and its Applications, 258(3):273-302, 1998.

[8] W. Fong and E. Darve. The black-box fast multipole method. Journal of Computational Physics, 228(23):8712-8725, 2009.

[9] A. Frangi. A fast multipole implementation of the qualocation mixed-velocity-traction approach for exterior Stokes flows. Engineering Analysis with Boundary Elements, 29(11):10391046, 2005.

[10] Y. Fu, K. J. Klimkowski, G. J. Rodin, E. Berger, J. C. Browne, J. K. Singer, R. A. Van De Geijn, and S. K. Vemaganti. A fast solution method for three-dimensional many-particle problems of linear elasticity. International Journal for Numerical Methods in Engineering, 42(7):1215-1229, 1998. 
[11] Y. Fu and G. J. Rodin. Fast solution method for three-dimensional Stokesian many-particle problems. Communications in Numerical Methods in Engineering, 16(2):145-149, 2000.

[12] Z. Gimbutas and L. Greengard. STFMMLIB3 - Fast Multipole Method (FMM) library for the evaluation of potential fields governed by the Stokes equations in $R^{3}$. http://www. cims. nyu.edu/cmcl/fmm3dlib/fmm3dlib.html, 2012.

[13] N. A. Gumerov and R. Duraiswami. Fast multipole method for the biharmonic equation in three dimensions. Journal of Computational Physics, 215(1):363-383, 2006.

[14] J. Happel and H. Brenner. Low Reynolds number hydrodynamics: with special applications to particulate media. Springer Science \& Business Media, 1983.

[15] S. Kim and S. J. Karrila. Microhydrodynamics: principles and selected applications. Butterworth-Heinemann, London, 1991.

[16] H. Lamb. Hydrodynamics. Cambridge University Press, 1932.

[17] R. D. Mindlin. Force at a point in the interior of a semi-infinite solid. Journal of Applied Physics, 7(5):195-202, 1936.

[18] H. Neuber. Ein neuer Ansatz zur Lösung räumlicher Probleme der Elastizitätstheorie. Der Hohlkegel unter Einzellast als Beispiel. ZAMM-Journal of Applied Mathematics and Mechanics/Zeitschrift für Angewandte Mathematik und Mechanik, 14(4):203-212, 1934.

[19] P. F. Papkovich. Solution Générale des équations differentielles fondamentales d'élasticité exprimée par trois fonctions harmoniques. Comptes Rendus de l'Académie des Sciences, 195:513$515,1932$.

[20] C. Pozrikidis. Boundary integral and singularity methods for linearized viscous flow. Cambridge University Press, 1992.

[21] S. E. Spagnolie and E. Lauga. Hydrodynamics of self-propulsion near a boundary: predictions and accuracy of far-field approximations. Journal of Fluid Mechanics, 700:105-147, 2012.

[22] A. K. Tornberg and L. Greengard. A fast multipole method for the three-dimensional Stokes equations. Journal of Computational Physics, 227(3):1613-1619, 2008.

[23] S. K. Veerapaneni, A. Rahimian, G. Biros, and D. Zorin. A fast algorithm for simulating vesicle flows in three dimensions. Journal of Computational Physics, 230(14):5610-5634, 2011.

[24] H. Wang, T. Lei, J. Li, J. Huang, and Z. Yao. A parallel fast multipole accelerated integral equation scheme for 3D Stokes equations. International Journal for Numerical Methods in Engineering, 70(7):812-839, 2007.

[25] H. T. Wang and Z. H. Yao. A new fast multipole boundary element method for large scale analysis of mechanical properties in 3D particle-reinforced composites. Computer Modeling in Engineering and Sciences, 7(1):85-95, 2005.

[26] X. Wang, J. Kanapka, W. Ye, N. R. Aluru, and J. White. Algorithms in FastStokes and its application to micromachined device simulation. Computer-Aided Design of Integrated Circuits and Systems, IEEE Transactions on, 25(2):248-257, 2006. 
[27] L. Ying, G. Biros, and D. Zorin. A kernel-independent adaptive fast multipole algorithm in two and three dimensions. Journal of Computational Physics, 196(2):591-626, 2004.

[28] K. Yoshida, N. Nishimura, and S. Kobayashi. Application of fast multipole Galerkin boundary integral equation method to elastostatic crack problems in 3D. International Journal for Numerical Methods in Engineering, 50(3):525-547, 2001.

[29] H.Y. Yu. Fundamental singularities in a two-fluid Stokes flow with a plane interface. Journal of Mechanics, 19(1):263-270, 2003. 